\title{
Körperstereotaxie und Lobektomie im randomisierten Vergleich beim NSCLC Stadium I - endlich Level-I-Evidenz?
}

\author{
Matthias Guckenberger · Nicolaus Andratschke
}

Online publiziert: 12 . Oktober 2015

(C) Springer-Verlag Berlin Heidelberg 2015

Hintergrund In drei randomisierten Studien sollte bei Patienten mit einem nicht-kleinzelligen Lungenkarzinom (NSCLC) im Stadium I der Therapiestandard, bestehend aus Lobektomie und Lymphknotendissektion, mit der Körperstereotaxie (SBRT) verglichen werden. Alle drei Studien mussten aufgrund mangelnder Rekrutierung vorzeitig geschlossen werden. In der aktuellen Arbeit werden die gepoolten Daten von zwei dieser Phase-III-Studien (ROSEL- und STARS-Studie) ausgewertet.

Methodik In der ROSEL- sowie der STARS-Studie wurden Patienten mit einem NSCLC im Stadium I $(<4 \mathrm{~cm})$ randomisiert im Verhältnis 1:1 entweder mit SBRT oder Lobektomie und mediastinaler Lymphknotendissektion behandelt.

Ergebnisse Insgesamt konnten die Daten von 58 Patienten ausgewertet werden, 31 mit SBRT und 27 mit Lobektomie. Nach einer medianen Nachbeobachtungszeit von 35-40 Monaten war das 3-Jahres-Gesamtüberleben nach SBRT signifikant besser als das nach Lobektomie (95 vs. $79 \%$; $p=0,037)$. Im Rezidivmuster wurden keine signifikanten Unterschiede beobachtet, nach SBRT allerdings fünf lokoregionäre Rezidive, nach Lobektomie nur eins. Wäh-

Originalpublikation Chang JY, Senan S, Paul MA et al (2015) Stereotactic ablative radiotherapy versus lobectomy, for operable stage I non-small-cell lung cancer: a pooled analysis of two randomised trials. Lancet Oncol 16:630-637

Prof. Dr. M. Guckenberger, M.D. ( $₫) \cdot$ Dr. N. Andratschke Department of Radiation Oncology,

University Hospital Zurich (USZ),

Rämistrasse 100,

8091 Zurich, Schweiz

E-Mail: Matthias.Guckenberger@usz.ch rend $44 \%$ der Patienten nach Lobektomie eine Toxizität vom Grad 3-4 erlitten, wurde dies nach SBRT nur in 10\% beobachtet.

Schlussfolgerung der Autoren Bei Patienten mit einem NSCLC im Stadium I stellt die SBRT eine alternative Behandlungsoption zur Lobektomie dar. Sie ist besser verträglich und resultiert in einem besseren Gesamtüberleben. Zukünftige Studien müssten diese Frage weiter untersuchen.

\section{Kommentar}

„Nichts wird oft so unwiederbringlich versäumt wie eine Gelegenheit" (Marie Ebner-Eschenbach). Nachdem die drei randomisierten Studien zum Vergleich von SBRT mit Lobektomie beim NSCLC im Stadium I nach der Rekrutierung kleinster Patientenzahlen abgebrochen wurden, muss den Autoren zu dieser gepoolten Analyse gratuliert werden. Die zahlreichen Leserbriefe an Lancet Oncology, vor allem von Thoraxchirurgen, lassen die hohe Brisanz der Publikation erkennen. Trotzdem müssen wir auf Schwachstellen und berechtigte Kritik an der Publikation hinweisen:

Die ROSEL- und STARS-Studien waren im Design zwar sehr ähnlich, aber eben nicht identisch, insbesondere was Einschlusskriterien, Dosierung und Fraktionierung der SBRT anging. Die Studiencharakteristika sind in der Tab. 1 zusammengefasst.

Die Autoren haben die statistischen Unsicherheiten der geringen Fallzahl von 58 Patienten diskutiert, die insbesondere auch angesichts der Zahl von 38 teilnehmenden Zentren kritisch gesehen werden muss. Obwohl das randomisierte Studiendesign den Selektion-Bias minimieren sollte, ist ein solcher angesichts der niedrigen Fallzahlen wohl nicht auszuschließen. So betrug das 3-Jahres-Über- 
Tab. 1 Vergleich der Studiencharakteristika von STARS und ROSEL

\begin{tabular}{|c|c|c|}
\hline & STARS & ROSEL \\
\hline Technologie & Cyberknife & C-Arm-Beschleuniger \\
\hline Diagnosesicherung & $\begin{array}{l}\text { Bioptische Siche- } \\
\text { rung notwendig }\end{array}$ & $\begin{array}{l}\text { Klinische Diagno- } \\
\text { se basierend auf } \\
\text { Bildgebung möglich }\end{array}$ \\
\hline Tumorlokalisation & Peripher: $3 \times 18$ Gy & Peripher: $3 \times 18$ Gy \\
\hline und Fraktionierung & Zentral: $4 \times 12,5$ Gy & Zentral: ausgeschlossen \\
\hline Stratifizierung & $\begin{array}{l}\text { Zentrum, } \\
\text { Tumorlokalisation, } \\
\text { Tumordurchmesser }\end{array}$ & $\begin{array}{l}\text { Zentrum, Histologie, } \\
\text { WHO-Performance- } \\
\text { Status }\end{array}$ \\
\hline $\begin{array}{l}\text { Teilnehmende } \\
\text { Zentren }\end{array}$ & $N=28$ & $N=10$ \\
\hline $\begin{array}{l}\text { Eingeschlossene } \\
\text { Patienten }\end{array}$ & $N=36$ & $N=22$ \\
\hline
\end{tabular}

leben nach SBRT $100 \%$ in der STARS-Studie und $89 \%$ in der ROSEL-Studie. Nach Lobektomie betrug das 3-JahresGesamtüberleben nur $67 \%$ in der STARS-, aber $100 \%$ in der ROSEL-Studie. Daher ist die Schlussfolgerung, dass eine SBRT das Gesamtüberleben mehr verbessert als eine Lobektomie zwar formal korrekt ( $p=0,037)$, eine vorsichtigere Formulierung wäre aber unseres Erachtens angebracht gewesen, z. B.: Nach SBRT ist das Gesamtüberleben nicht schlechter als nach Lobektomie.

Ungerechtfertigte Kritik an der Publikation Die Auswertung basiert auf zwei randomisierten Studien mit jeweils exzellenter Qualitätssicherung und der aktuelle chirurgische Therapiestandard der offenen Lobektomie war ebenso erfüllt wie der aktuelle radioonkologische Therapiestandard der SBRT. Ob durch die konsequente Anwendung von VATS der eklatante Unterschied in der Grad-3- und -4-Toxizität (10\% nach SBRT gegenüber $44 \%$ nach Lobektomie) hätte vermindert oder sogar ausgeglichen werden, darf bezweifelt werden. Immerhin 5/27 operierten Patienten wurden mittels VATS behandelt, und auch nach VATS-Lobektomie erleiden immer noch fast 30\% der Patienten postoperative Komplikationen [1]. Lediglich ein Patient verstarb an den Folgen des operativen Eingriffs, was auf eine gute und repräsentative Qualität der Thoraxchirurgie rückschießen lässt. Umgekehrt hat sich seit dem Design der beiden Studien auch die Praxis der SBRT weiterentwickelt: Es kann spekuliert werden, dass durch den Einsatz der 4-D-Bildgebung und 4-D-Image-Guidance, der VMAT-Bestrahlungsplanung und FFF-Behandlungsapplikation die Ergebnisse der SBRT noch weiter hätten verbessert werden können.

Neuartigkeit der Publikation In vielerlei Hinsicht berichtet die aktuelle Publikation keine Erkenntnisse und Daten, die nicht schon identisch oder zumindest ähnlich bekannt waren. In zahlreichen Matched-Pair- und Propensity-Machted-Analysen wurde die SBRT mit Lobektomie, offen oder VATS, mit Segmentektomie oder Keilresektion verglichen.
Die Analysen sind alle konsistent im Ergebnis, nämlich dass die SBRT kein signifikant schlechteres Überleben als die chirurgischen Verfahren erzielt, allerdings bei besserer Verträglichkeit [2]. Auffällig war die höhere Zahl an regionären Lymphknotenrezidiven nach SBRT $(n=4)$ im Vergleich mit der Lobektomie bei der mediastinalen Lymphknotendissektion $(n=1)$. Die Autoren diskutieren, ob durch den systematischen Einsatz von EBUS-Biopsien in cN0-Patienten zukünftig die Anzahl der regionären Rezidive nach alleiniger SBRT des Primarius reduziert werden kann. Dies muss prospektiv untersucht werden, insbesondere auch, ob die erforderlich hohe EBUS-Qualität in spezialisierten Zentren auf die breite Anwendung übertragbar ist. Und im Übrigen, selbst in chirurgischen Studien konnte die Notwendigkeit der systematischen Lymphknotendissektion quo ad vitam nicht bewiesen werden [3].

Erklärungsmodelle der unerwarteten Ergebnisse Wenn wir die Zahlen und Ergebnisse der Arbeit akzeptieren, dann gilt es zu fragen, warum die lokal radikalere Therapiemodalität (Lobektomie mit Lymphknotendissektion) zwar die lokoregionäre Kontrolle verbessert, aber nicht das Überleben. Hierfür gibt es verschiedene Erklärungsmodelle, die über einfaches Negieren hinausgehen. Zum einen wird in kleineren, weniger spezialisierten thoraxchirurgischen Einrichtungen eine schlechtere Ergebnisqualität erzielt als in großen Zentren. Dies betrifft sowohl die perioperative Letalität, die postoperativen Komplikationen als auch das Langzeitüberleben [4-6]. Das wurde für die SBRT interessanterweise bisher nicht gezeigt: In nationalen Patterns-of-Care- und Registerstudien liegt die Letalität nach SBRT konsistent bei $<1 \%$ und die lokale Tumorkontrolle bei $\geq 90 \%$, wenn die Bestrahlung mit ausreichender Intensität durchgeführt wird [7, 8]. Eine bessere Standardisierbarkeit und sichere Implementierung der Strahlentherapie auf hohem Niveau ,in der Breite“ könnte folglich zumindest teilweise die sehr guten Ergebnisse der SBRT erklären. Zweitens wissen wir, dass sowohl SBRT als auch Lobektomie erfolgreiche lokale Therapieverfahren darstellen und die Mehrzahl der Rezidive distant auftreten [9, 10]. Ob vor diesem Hintergrund eine weitere Intensivierung der lokalen Therapie über das bisherige Niveau der SBRT hinaus die Überlebenschancen tatsächlich weiter verbessern kann oder ob nicht zusätzliche Toxizität den potentiellen Benefit wieder zunichtemacht, ist durchaus zu bedenken [11], muss aber weiterhin untersucht werden.

\section{Fazit}

Bei Patienten mit einem NSCLC im Stadium I stellt die SBRT eine alternative Behandlungsoption zur Lobektomie dar. Sie ist besser verträglich und resultiert bezüglich des 
Gesamtüberlebens in ähnlichen Ergebnissen. Wie so oft am Ende einer Diskussion wird auf die Notwendigkeit weiterer randomisierter Studien hingewiesen. Dies ist auch im aktuellen Fall sicher korrekt, zumal tatsächlich weitere solcher Studien in Vorbereitung sind. Ob solche Studien allerdings erfolgreicher rekrutieren werden als die bisherigen, bleibt abzuwarten. Gerade nach der Publikation der hier kommentierten Studie wird das nicht einfacher werden. Auch das sehr unterschiedliche Nebenwirkungsprofil der beiden Therapieoptionen könnte Patienten von einer Teilnahme abhalten. Auf absehbare Zeit werden wir folglich mit der aktuell verfügbaren Evidenz leben müssen. Diese erlaubt es noch nicht, beim NSCLC im Stadium I die SBRT als neuen Therapiestandard zu deklarieren. Aber alle Patienten müssen über die Möglichkeit der SBRT informiert werden; und das muss interdisziplinär erfolgen. Dieser Aufgabe müssen auch wir uns als Radioonkologen stellen. Die hier kommentierte Arbeit ist ein wichtiges Glied in der Argumentationskette, aber auch nur dann, wenn wir sie korrekt zu interpretieren wissen.

\section{Matthias Guckenberger und Nicolaus Andratschke, Zürich}

\section{Literatur}

1. Scott WJ, Allen MS, Darling G, Meyers B, Decker PA, Putnam JB, McKenna RW, Landrenau RJ, Jones DR, Inculet RI et al (2010) Video-assisted thoracic surgery versus open lobectomy for lung cancer: a secondary analysis of data from the American College of Surgeons Oncology Group Z0030 randomized clinical trial. J Thorac Cardiovasc Surg 139:976-981. (discussion 981-973)

2. Guckenberger M (2015) Stereotactic body radiotherapy in operable patients with stage I NSCLC: where is the evidence? Expert Rev Anticancer Ther 15(5):525-530. doi:10.1586/14737140.2015.1023190
3. Darling GE, Allen MS, Decker PA, Ballman K, Malthaner RA, Inculet RI, Jones DR, McKenna RJ, Landreneau RJ, Rusch VW et al (2011) Randomized trial of mediastinal lymph node sampling versus complete lymphadenectomy during pulmonary resection in the patient with N0 or N1 (less than hilar) non-small cell carcinoma: results of the American College of Surgery Oncology Group Z0030 Trial. J Thorac Cardiovasc Surg 141:662-670

4. Bach PB, Cramer LD, Schrag D, Downey RJ, Gelfand SE, Begg CB (2001) The influence of hospital volume on survival after resection for lung cancer. N Engl J Med 345:181-188

5. Cheung MC, Hamilton K, Sherman R, Byrne MM, Nguyen DM, Franceschi D, Koniaris LG (2009) Impact of teaching facility status and high-volume centers on outcomes for lung cancer resection: an examination of 13,469 surgical patients. Ann Surg Oncol $16: 3-13$

6. Luchtenborg M, Riaz SP, Coupland VH, Lim E, Jakobsen E, Krasnik M, Page R, Lind MJ, Peake MD, Moller H (2013) High procedure volume is strongly associated with improved survival after lung cancer surgery. J Clin Oncol 31:3141-3146

7. Guckenberger M, Allgauer M, Appold S, Dieckmann K, Ernst I, Ganswindt U, Holy R, Nestle U, Nevinny-Stickel M, Semrau S et al (2013) Safety and efficacy of stereotactic body radiotherapy for stage i non-small-cell lung cancer in routine clinical practice: a patterns-of-care and outcome analysis. J Thorac Oncol 8:1050-1058

8. Shirvani SM, Jiang J, Chang JY, Welsh JW, Gomez DR, Swisher S, Buchholz TA, Smith BD (2012) Comparative effectiveness of 5 treatment strategies for early-stage non-small cell lung cancer in the elderly. Int J Radiat Oncol Biol Phys 84:1060-1070

9. Su S, Scott WJ, Allen MS, Darling GE, Decker PA, McKenna RJ, Meyers BF (2014) Patterns of survival and recurrence after surgical treatment of early stage non-small cell lung carcinoma in the ACOSOG Z0030 (ALLIANCE) trial. J Thorac Cardiovasc Surg 147:747-752. (Discussion 752-743)

10. Senthi S, Lagerwaard FJ, Haasbeek CJ, Slotman BJ, Senan S (2012) Patterns of disease recurrence after stereotactic ablative radiotherapy for early stage non-small-cell lung cancer: a retrospective analysis. Lancet Oncol 13:802-809

11. Zhang J, Yang F, Li B, Li H, Liu J, Huang W, Wang D, Yi Y, Wang $\mathrm{J}$ (2011) Which is the optimal biologically effective dose of stereotactic body radiotherapy for Stage I non-small-cell lung cancer? A meta-analysis. Int J Radiat Oncol Biol Phys 81:e305-e316 